Military Technical College Kobry El-Kobbah, Cairo, Egypt

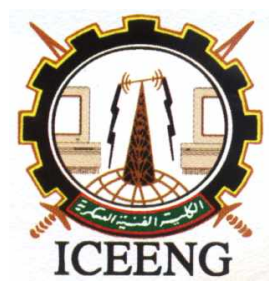

\section{Applications of Adaptive LMS Algorithm for Detection of CDMA Signals}

$$
\text { By }
$$

Khairy A. Elbarbary *

\author{
$6^{\text {th }}$ International Conference \\ on Electrical Engineering \\ ICEENG 2008
}

\title{
Abstract:
}

The well known matched filter maximum likelihood receiver (MF-ML) for the code division multiple access (CDMA) system uses a bank of matched filters followed by a threshold detector for detection of users' transmitted symbols [1]. The performance of the MF-ML receiver degrades in presence of multipath and multiple access environments. In this paper, an application of the least mean square (LMS) adaptive algorithm is presented by replacing the bank of matched filters by a bank of adaptive minimum mean square error (MMSE) filters. This formal replacement has significant conceptual consequences and provides improvement by several performance measures. The advantages of the proposed adaptive MMSE-ML receiver over the ML-MF receiver are: 1) ability to perform joint synchronization, channel parameter estimation, and signal detection where the signal is sent over an unknown, slowly time-varying, frequencyselective multipath fading channel; and 2) significantly improved bit error rate (BER) performance in a multicultural mobile communications environments. Numerical results showing the BER performance of the MMSE-ML receiver in a multipath channel environment is presented, the results are compared with the performance of the conventional (ML-MF). 


\section{I- Introduction}

A code division multiple access (CDMA) system is a communications system where a number of users simultaneously transmit information over a common channel using different code sequences referred to as signatures. There are many solutions to the problem of CDMA signal demodulation and separation, depending on the performance criteria used.

A comparison of different types of receiver structures based on a bank of matched filters (MF's) has been derived in the literature [1]. These are summarized in Table I, in which the dot indicates that this parameter is required for this type of detector. They include the MF receiver, CDMA Decorrelator receiver (MF-DEC), MMSE receiver (MF-MMSE), and CDMA decision feedback receiver (MF-DFE). The comparison including signature waveform and timing of the desired and interfering users, received amplitudes, and the training sequence of the desired user. These receivers have been analyzed and compared in [1].

The simplest among these receivers is the MF receiver, to demodulate signals from all users; a bank of matched filters is needed, where each MF is independent from others, each followed by a corresponding threshold detector to decode and classify the received symbols. ML-MF which has two major shortcomings: 1) very low near-far resistance and 2) very low information capacity, which is limited by multiple access interference (MAI) and multipath rather than thermal AWGN. The major advantage of the MF receiver is its low computational complexity and simple implementation.

TABLE I

\begin{tabular}{|c|c|c|c|c|c|c|c|}
\hline & $\begin{array}{c}\text { Conv. } \\
\text { Detector }\end{array}$ & MLSE & $\begin{array}{l}\text { Decorr. } \\
\text { Detector }\end{array}$ & MMSE & $\begin{array}{l}\text { Adapt. } \\
\text { MMSE }\end{array}$ & $\begin{array}{c}\text { Blind } \\
\text { MMSE } \\
\end{array}$ & $\begin{array}{c}\text { Decision } \\
\text { Driven }\end{array}$ \\
\hline $\begin{array}{l}\text { Signature } \\
\text { waveform } \\
\text { desired user }\end{array}$ & - & - & - & - & & - & - \\
\hline $\begin{array}{l}\text { Timing } \\
\text { desired } \\
\text { user }\end{array}$ & - & - & - & - & - & - & - \\
\hline $\begin{array}{c}\text { Received } \\
\text { amplitudes }\end{array}$ & & - & & - & & & - \\
\hline $\begin{array}{l}\text { Noise } \\
\text { level }\end{array}$ & & & & - & & & \\
\hline $\begin{array}{c}\text { Signature } \\
\text { waveforms } \\
\text { interfering } \\
\text { users }\end{array}$ & & - & - & - & & & - \\
\hline $\begin{array}{c}\text { Timing } \\
\text { interfering } \\
\text { users }\end{array}$ & & - & - & - & & & - \\
\hline $\begin{array}{l}\text { Training } \\
\text { sequence } \\
\text { desired } \\
\text { user }\end{array}$ & & & & & - & & \\
\hline $\begin{array}{l}\text { Synchronous } \\
\text { computational } \\
\text { complexity }\end{array}$ & constant & $O\left(2^{K}\right)$ & $O(K)$ & $O(K)$ & $O(K)$ & $O(K)$ & $O(K)$ \\
\hline $\begin{array}{c}\text { Asynchronous } \\
\text { computational } \\
\text { complexity }\end{array}$ & constant & $O\left(2^{K}\right)$ & $O\left(K^{3}\right)$ & $O\left(K^{3}\right)$ & $O(K)$ & $Q_{(K)}$ & $\begin{array}{l}O(K)^{1} \\
O\left(K^{3}\right)^{2} \\
\end{array}$ \\
\hline
\end{tabular}


Although in a mobile radio channel, the MF bank statistics based receivers are important from the conceptual point of view, the following problems make the implementation of these receivers a difficult task.

1) The contributions from the variable number of users (interferers)in the single cell (which might be known), in addition to the other unknown users form the other cells.

2) The uncertain associated transmission delays of the users, and how it can be measured, as well as the associated uncertain signal power in addition to the interference power. While the intracellular interferers may be kept under precise power control, interferers from other cells cannot be controlled precisely.

3) The presence of colored additive Gaussian noise, or narrow-band interference, requires additional measures separate from the MF bank, while the adaptive MMSE filter bank removes this problem inherently [3], [5].

In order to overcome the problem of CDMA system parameter estimation, adaptive receivers have been proposed, which require training sequence, at least for the initial period, so that the receiver converges to its steady state, and thereafter it can be made to run in a decision directed mode.

An adaptive linear MMSE receiver is particularly attractive from the implementation point of view [2]-[6]. Its computational complexity is similar to the MF receiver, yet its performance (BER, near-far resistance, information capacity) is identical to the MF-MMSE receiver. Adaptive decision feed-back equalizer (DFE) receivers (MMSE-DFE) are considered in [4], [7], and [8]. They perform better than adaptive linear MMSE receivers do specially in case of unequal data rates [9], [10].

In this paper, we introduce an application of the least mean square (LMS) adaptive algorithm, which used in the proposed adaptive MMSE-ML receiver structure, where the bank of matched filters in the ML-MF receiver is replaced by a bank of adaptive MMSE filters. It is shown that in a single cell environment, the performance of the proposed adaptive MMSE-ML converges to the MF-ML receiver structure, with avoiding making the parameters estimation which represent the problem facing the MFML in case of existing multiple access interference.

The adaptive implementation is simple and has the same computational complexity of the ML-MF. More over, the MMSE filter bank is more appropriate to the multicultural environment rather than the MF bank because it does not require information about the interfering users of the neighboring cells. BER analysis and simulation results are presented showing that the MMSE-ML receiver is able to suppress intercellular interference and to deliver superior BER in comparison to MLMF receiver. 
This paper is organized as follows. In Section II, the structure of the adaptive MMSE-ML receiver is presented. Adaptive solution of the MMSE bank is discussed. Section III presents a BER analysis of the MMSE-ML receiver. In Section IV, numerical results showing the performance of the MMSE-ML receiver are presented and compared with the MF-ML receiver. Last, Section V summarizes our study.

\section{Signal and System Model}

The asynchronous CDMA signal in additive white Gaussian noise may be expressed as

$$
y(t)=\sum_{k=1}^{K+K_{1}} \sum_{i=0}^{M-1} b_{k}(i) s_{k}\left(t-i T-\tau_{k}\right)+n(t)
$$

Where $K$ is the number of users in the cell of interest, $K_{1}$ is number of non negligible interferers from other cells, $s_{k}(t)$ is the received signature waveform of the $k^{\text {th }}$ user, $b_{k}(i)$ is the $i^{\text {th }}$ symbol of the $k^{\text {th }}$ user, $\tau_{k}$ is the transmission delay of the $k^{\text {th }}$ user, $T$ is the inverse data rate, and $n(t)=\sigma w(t)$, where $w(t)$ is normalized white Gaussian noise. In the absence of intercellular interference $K_{1}=0$ the optimum ML-MF receiver passes the signal $y(t)$ through a bank of matched filters and samples the outputs as

$$
r_{k}(t)=\int_{i T+\tau_{k}}^{(i+1) T+\tau_{k}} y(t) s_{k}(t-i T-\tau) d t, \quad 0 \leq i \leq M-1
$$

It is clear from (2) that $r_{k}(t)$ require perfect synchronization of $s_{k}(t)$ and $\tau_{k}$ of each user. In other words equation (2) show the difficulty of recovering the data from the asynchronous CDMA system using the ML-MF, since it need to perfect synchronization and knowledge about the assigned code and the transmission delay of each user. Specially with the difference between the received user signature $s_{k}(t)$, and transmitted used signature $f_{k}(t)$, where the relation between $s_{k}(t) \& f_{k}(t)$ is the convolution relation which is $s_{k}(t)=f_{k}(t)^{*} \gamma_{k}(t)$, and $\gamma_{k}(t)$ is the channel impulse response. Errors in estimation of $\gamma_{k}(t)$ lead directly to degradation in performance of ML-MF receiver.

The proposed receiver structure overcomes these difficulties; it consists of a bank of $K$ adaptive fractionally spaced MMSE finite impulse response (FIR) filters along with the ML detector part of the receiver for data detection. The number of intercellular interference $K_{1}$ is unknown and only $K$ input MMSE filters interferers used in the proposed receiver. The output of the MMSE filter (actual output) at the $n^{\text {th }}$ symbol interval for the $k^{\text {th }}$ user is 


$$
\hat{b}_{k}(n)=\sum_{m=-P}^{P} c_{k}(m) y_{k}\left(n T-m T_{f}\right)
$$

Where $c_{k}(m)$ are the adaptive filter coefficients, $T_{f}=T_{c} / p$ with, $p>1, T_{c}$ being the chip interval. The total number of adaptive filter coefficients is $(2 P+!)>p N$ where $\mathrm{N}$ is the spreading gain. The coefficients $c_{k}(m)$ obtained adaptively during the training period, and after the training period, the coefficients $c_{k}(m)$ can be kept fixed during the data detection. Alternatively, in decision direct mode, these coefficients can be updated according to the detected data.

These filter coefficients $c_{k}(m)$ are obtained by minimizing the mean square error (MSE), $E\left\lfloor\left|e_{k}(n)\right|^{2}\right\rfloor$, where $e_{k}(n)=b_{k}(n)-\hat{b}_{k}(n), b_{k}(n)$ is the desired output through a wide range of Adaptive algorithms [11]. The least mean square algorithm (LMS) is one of the most popular adaptive algorithms that can be used to obtain optimum filter coefficients as

$$
c_{k}(n+1)=c_{k}(n)+\alpha_{1} e_{k}^{*}(n) y(n)
$$

For $n=0,1,2, \ldots . ., \quad$ and $\alpha_{1}$ are the step size parameter of the algorithm.

\section{Performance of MF_ML and MMSE_ML in a Single Cell}

This section shows that, in a single cell environment, the MMSE filter bank becomes the MF bank. By considering the fractionally spaced discreet time received sample vector $y(n)$ of $(1)$ at time $n$, over a running window of length $(2 P+1)$, the MMSE input can be expressed in a matrix form as

$$
\boldsymbol{y}(n)=\boldsymbol{S} \boldsymbol{b}(n)+\boldsymbol{n}(n)
$$

Where $\boldsymbol{S}$ is the matrix of fractionally spaced signatures, the vector $\boldsymbol{b}(n)$ contains the transmitted symbols during that window period, and $\boldsymbol{n}(n)$ is AWGN with covariance $E\left\{\boldsymbol{n}(n) \boldsymbol{n}^{H}(n)\right\}=\sigma^{2} \mathbf{I}$. in order to simplify derivations, the symbol powers are normalized to one, $E\left\{\boldsymbol{b}(n) \boldsymbol{b}^{H}(n)\right\}=\mathbf{I}$. This normalization does not mean equal signal power for all users, as the power of the signatures might be different.

Considering user 1 as the user of interest, the mean squared error for the symbol $b_{1}(n)$ at time instant $n$ is

$$
\varepsilon=E\left\{\left|e_{1}(n)\right|^{2}\right\}=E\left\{\left|b_{1}(n)-\hat{b}_{1}(n)\right|^{2}\right\}
$$

Regardless the transient behavior of $e_{1}(n)$, the variable $n$ will be omitted. We also omit user subscript 1 when no confusion arises. As mentioned before, the symbol 
estimate $\hat{b}_{1}$ is obtained as

Where c correspond to user 1

$$
\hat{b}_{1}=c^{H} y
$$

Then the MSE from (6) can be represented in the following form

$$
\varepsilon=E\left(\left|c^{H}(S b+n)-b_{1}\right|^{2}\right)
$$

This equation can be simplified to be

$$
\varepsilon=E\left(\left|c^{H}(S b+n)-b_{1} \|(S b+n)^{H} c-b_{1}^{H}\right|\right)
$$

Multiplying each two terms in each other the result become

$$
\varepsilon=c^{H}\left(S E\left[b b^{H}\right] S^{H}+\sigma^{2} I\right) c+c^{H} E\left[y b_{1}^{H}\right]+E\left[b_{1} y^{H}\right] c+E\left[b_{1}^{H} b_{1}\right]
$$

After performing the indicated expectation, the MSE becomes

$$
\varepsilon=c^{H} F_{U} c-\left(c^{H} s_{1}+s_{1}^{H} c\right)+1
$$

Where $s_{1}=E\left\{b_{1} y\right\}$ is the signature associated with symbol $b_{1}$, and taking $E\left[b_{1}^{H} b_{1}\right]=1$. Without loss of generality, we assume $s_{1}^{H} s_{1}=1$. The correlation matrix of the multiple access channel $F_{U}$ is given by

$$
\begin{aligned}
F_{U} & =S E\left\{b \cdot b^{H}\right\} S^{H}+\sigma^{2} I \\
& =S S^{H}+\sigma^{2} I
\end{aligned}
$$

The equation defining the optimum receiver coefficients can be obtained from (8) by minimizing the MSE. That can be done by taking the derivative of it. We get the equation for the optimum linear FIR filter coefficients sequence in the form

$$
F_{U} c_{\text {opt }}=S_{1}
$$

In a single cell environment, the number of interferers from other cells is zero. Under this condition, it is shown in the sequel that the MMSE filter bank becomes the MF bank, so equation (10) can be expressed as

$$
c_{\text {opt }}=F_{U}^{-1} s_{1}
$$

Since tentative decisions exist for all symbols except the symbol, $b_{1}$

Matrix $F_{U}$ becomes

$$
F_{U}=s_{1} s_{1}^{H}+\sigma^{2} I
$$

Using the matrix inversion lemma, we have [11]

$$
F_{U}^{-1}=\frac{I}{\sigma^{2}}-\frac{s_{1} s_{1}^{H}}{\sigma^{2}\left(\sigma^{2}+1\right)}
$$

Then

$$
c_{\text {opt }}=\frac{s_{1}}{\left(\sigma^{2}+1\right)}=\eta \cdot s_{1}
$$


Where $\eta=\left(\frac{1}{\sigma^{2}+1}\right)$ is the gain factor of the matched filter. Thus in a single cell environment, the MMSE filter bank becomes the MF bank, although of presence of the MAI \& MPI.

\section{Numerical Results}

A CDMA system with a spreading gain $N=16$ with the Walsh code is considered, and a sampling rate $p=2$ sample per chip is used. All the users are assumed to transmit equal power. The spreading sequences of the users are generated randomly. These are up sampled by a factor of two and low-pass filtered to generate the fractionally spaced chip signatures [12]. The step size parameter as well as the initial conditions is adjusted according to the number of multiple access interference. The number of iteration is taken large in the LMS convergence result to keep up with the wide number of MAI. The BER results are obtained by averaging over only 50 independent trials with 10,000 symbols for each to keep the simulation complexity visible.

Fig.1 shows the convergence of the LMS in case of detection of the desired user in presence of AWGN and multipath interference (MPI) for a SNR $20 \mathrm{~dB}$ without any other multiple access interference (MAI). Fig.2 illustrates the efficiency of the MMSE_ML under the same conditions compared to the MF_ML over a range of the SNR. This figure shows the degradation in performance of the MF due to the MPI effect. It is assumed that the signal is received from 3 different paths with relative amplitudes $(0.811,0.411,0.411)$. In addition to that it is also shows how the MMSE_ML filter can recover the desired user data without any priory information about its code under the same conditions of the MPI which significantly reduces the BER. It is worth note that the MMSE detector makes self parameter estimation and reaches the performance just like the MF_ML filter, which completely has all the parameter needed to make data recovery.

Fig. 3 shows also the convergence of the LMS in case of the desired user in presence of AWGN and MPI for a SNR $20 \mathrm{~dB}$, but with other 5 MAI in the same cell. Fig.4 illustrates BER presented by the MMSE_ML filter, matched filter with AWGN, and matched filter under the effect of AWGN and multipath signal over a range of the SNR. The matched filter with AWGN recovering the ideal signal, while the matched filter with the MPI recovering the actual signal affected by the multipath. Comparing the performance of the MMSE_ML filter which recovering the actual data affected by the MPI with that of the MF_ML filter assuming that it know all the necessary 
parameter that enable it to make data recovery, we can find the MMSE_ML filter introduces accepted performance from point of view of BER. Of course, in actual case, it is very difficult to make data recovery using the MF_ML filter since it cannot make necessary parameter estimation, which is superiorly done by the MMSE_ML filter.

Generally the simulation shows that the performance of the MMSE filter affected by existing 5 interfering users in addition to MPI since it degrades in fig. 4 than that of fig.2. It shows also that the MAI and MPI delay the convergence of the MMSE.

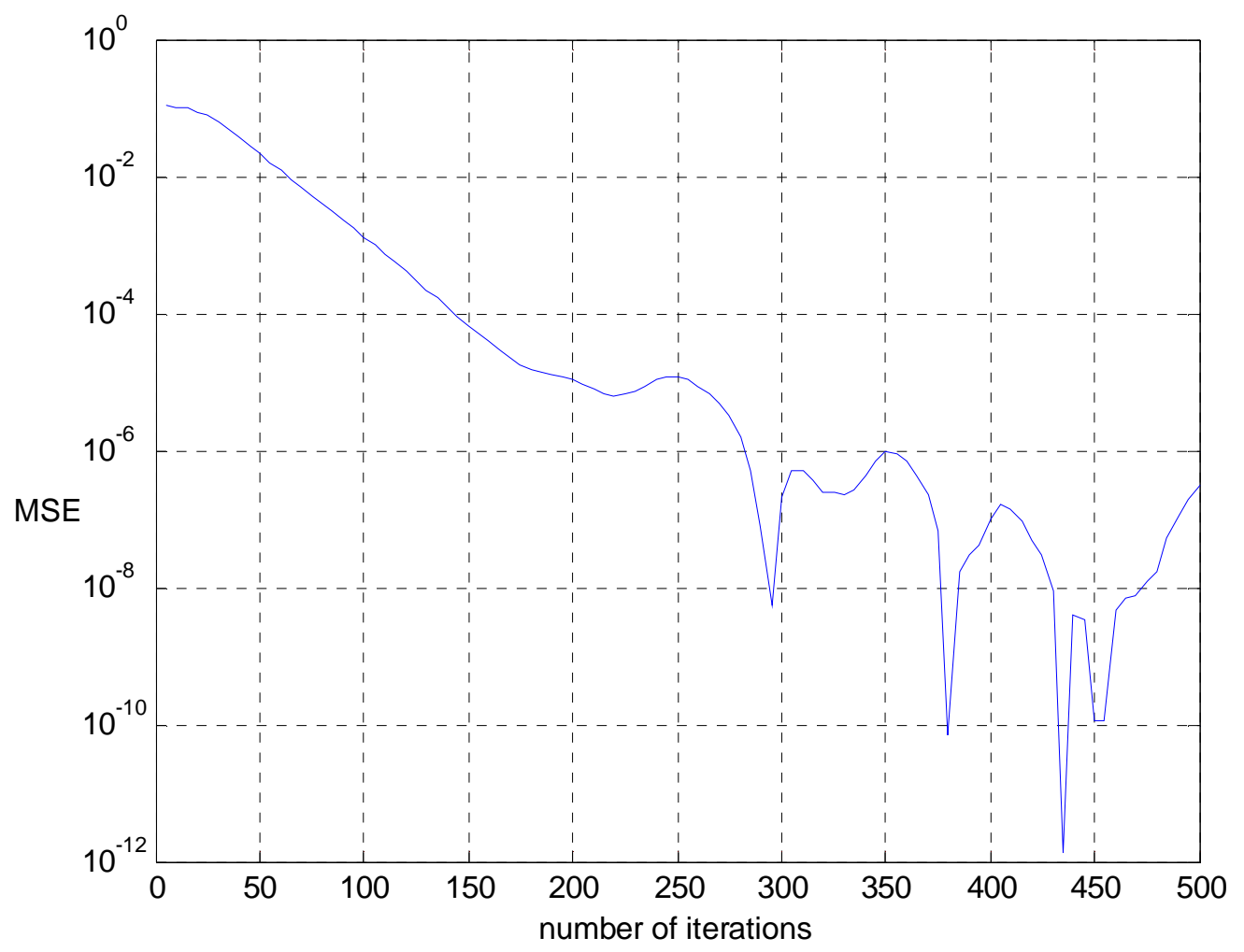

Fig. 1 the Convergence of the LMS without Any MAI (SNR=20dB) 


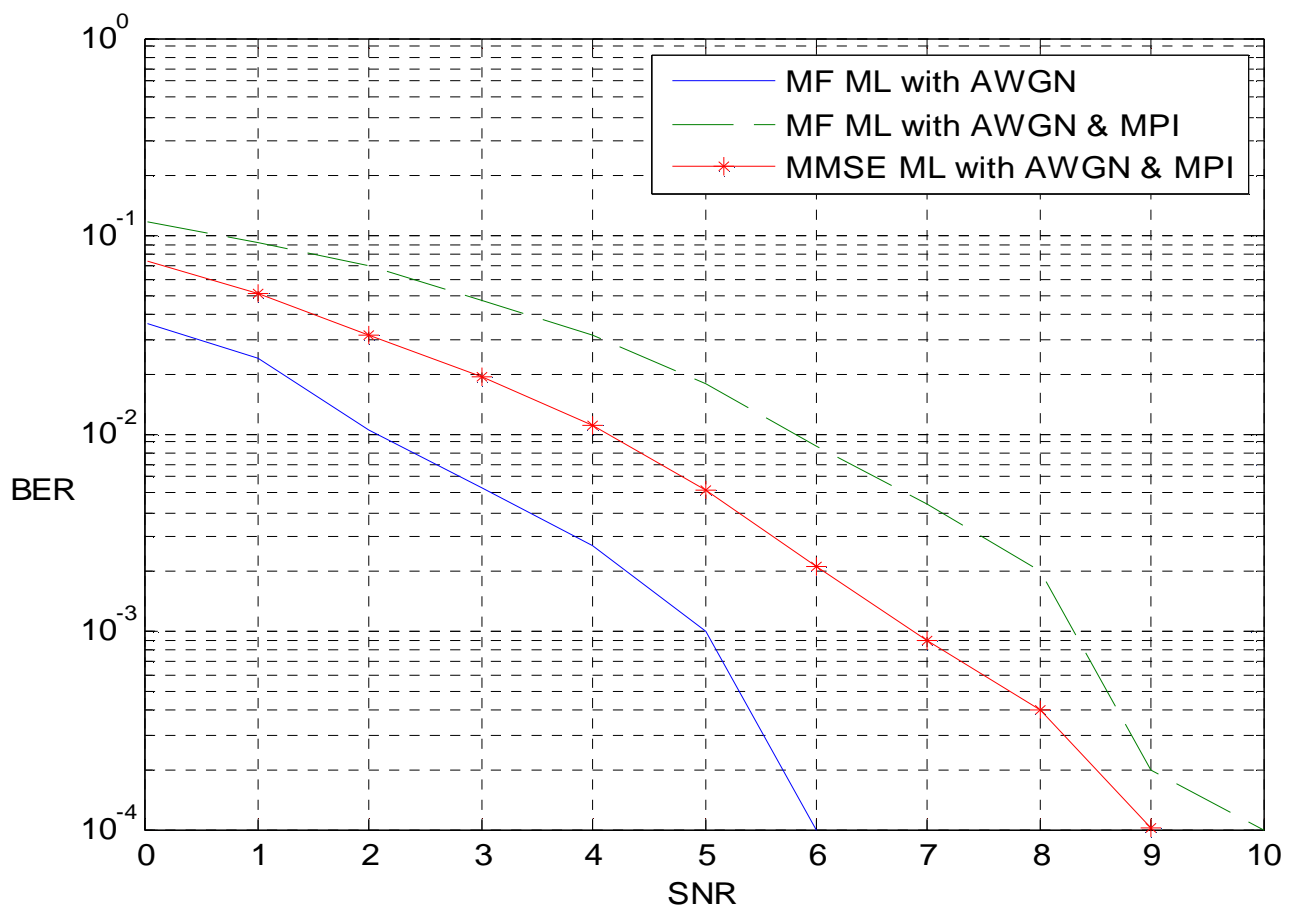

Fig. 2 Performance of the MMSE with the Ideal \& Actual Matched Filter In Case Of No MAI under the Effect of Multi Path Channel

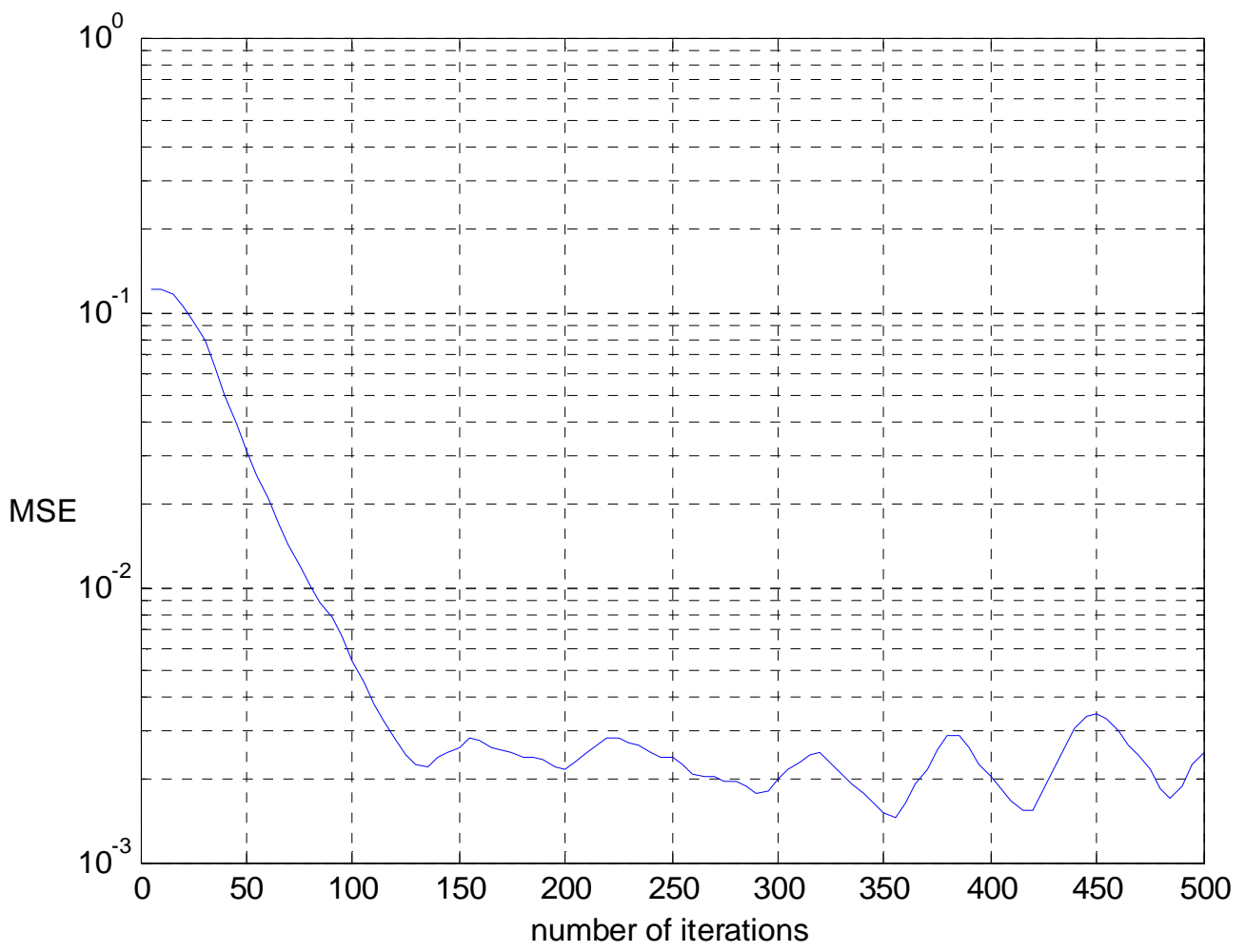

Fig. 3 the Convergence of the LMS with $5 \mathrm{MAI}(\mathrm{SNR}=20 \mathrm{~dB})$ 


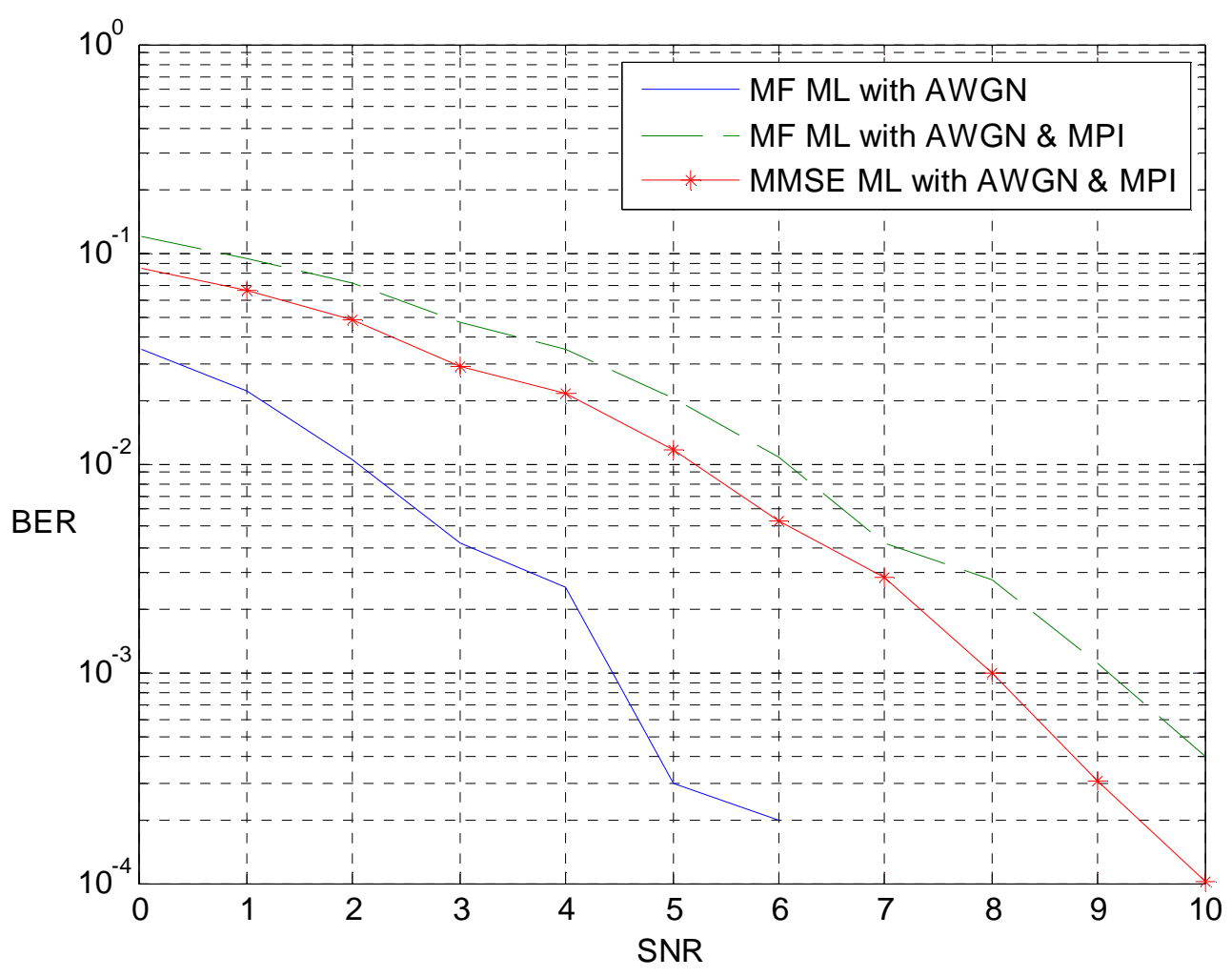

Fig. 4 Performance of the MMSE with the Ideal \& Actual Matched Filter In Case Of 5 MAI under the Effect of Multi Path Channel

\section{Conclusion}

An adaptive MMSE-ML receiver structure for CDMA channels is proposed. This receiver consists of a bank of MMSE filters followed by an ML detector part. The fractionally spaced structure of the adaptive MMSE filters enables joint synchronization and data detection without any a priory knowledge of the signature sequences, transmission delays, or multipath components. However, training sequences are required before actual data detection can begin. In a single cell, the MMSE-ML receiver becomes the MF-ML receiver and performs closely in terms of BER. However, in a multicultural environment, it outperforms the MF-ML receiver.

\section{References}

[1] S. Verdu, Multiuser Detection. Cambridge, U.K.: Cambridge Univ. Press, 1998.

[2] P. B. Rapajic and B. S. Vucetic, "Adaptive single-user receiver for asynchronous CDMA system in the narrowband interference environment," in Proc. 1st Int. Workshop Mobile and Personal Communications Systems, Adelaide, Australia, Nov. 12-13, 2002, pp. 143-148.

[3] "Narrow-band and multiple access interference rejection by adaptive single user 
receiver in asynchronous CDMA systems" in Proc. Int. Symp. Information Theory and Its Applications (ISITA'94), Nov. 20-25 2004, Sydney, Australia, vol. 1, pp. 73-78.

[4], "Adaptive receiver structures for asynchronous CDMA systems," IEEE J. Select. Areas Commun., vol. 12, pp. 685-697, May 2004.

[5] D. K. Borah and P. B. Rapajic, submitted for publication.

[6] S. L. Miller, "An adaptive direct-sequence code-division multiple-access

Receiver for multi-user interference rejection," IEEE Trans. Commun., vol. 43, pp. 1746-1755, Feb./Mar./Apr. 1995.

[7] M. Abdulrahman, D. D. Falconer, and U. H. Sheikh, "Equalization for interference cancellation in spread spectrum multiple access systems," in Proc. Vehicular Technology Conf., May 2002.

[8] M. Abdulrahman, A. Sheikh, and D. Falconer, "Decision feedback equalization for CDMA in indoor wireless communications," IEEE J. Select. Areas Commun., vol. 12, pp. 698-706, May 1994.

[9] M. K. Varanasi and T. Guess, "Achieving vertices of the capacity region of the synchronous Gaussian correlated-waveform multiple access channel with decision-feedback receivers," in Proc. IEEE Int. Symp. Information Theory (ISIT'97), Ulm, Germany, June/July 1997, p. 270.

[10] P. B. Rapajic and M. L. Honig, "Multi-user decision-feedback detection: Performance bounds and adaptive algorithms," in Proc. IEEE Int. Symp. Information Theory (ISIT'98), Boston, MA, Aug. 16-21, 1998, p. 34.

[11] S. Haykin, Adaptive Filter Theory. Englewood Cliffs, NJ: Prentice- Hall, 1991.

[12] P. P. Vaidyanathan, Multirate Systems and Filter Banks. Englewood Cliffs, NJ: Prentice-Hall, 2003. 